\title{
Music-supported therapy in the rehabilitation of subacute stroke patients: A randomized controlled trial.
}

\section{Grau-Sanchez, Jennifer}

\author{
2018-07
}

Grau-Sanchez , J , Ramos-Escobar , N , Sierpowska , J , Rueda , N , Susana , R , Rifà , X , Veciana de las Heras , M , Pedro , J , Särkämö , T T , Duarte , E \& Rodríguez-Fornells , A 2018 , ' Music-supported therapy in the rehabilitation of subacute stroke patients: A randomized controlled trial. ' , Annals of the New York Academy of Sciences, vol. 1423 , no. 1 , pp. 318-328 . https://doi.org/10.1111/nyas.13590

http://hdl.handle.net/10138/304268

https://doi.org/10.1111/nyas. 13590

unspecified

publishedVersion

Downloaded from Helda, University of Helsinki institutional repository.

This is an electronic reprint of the original article.

This reprint may differ from the original in pagination and typographic detail.

Please cite the original version. 


\title{
Music-supported therapy in the rehabilitation of subacute stroke patients: a randomized controlled trial
}

\author{
Jennifer Grau-Sánchez, ${ }^{1}$ Esther Duarte,${ }^{2}$ Neus Ramos-Escobar,${ }^{1}$ Joanna Sierpowska, ${ }^{1}$ \\ Nohora Rueda, ${ }^{2}$ Susana Redón, ${ }^{2}$ Misericordia Veciana de las Heras, ${ }^{3}$ Jordi Pedro, ${ }^{3}$ \\ Teppo Särkämö, ${ }^{4}$ and Antoni Rodríguez-Fornells ${ }^{1,5}$ \\ ${ }^{1}$ Cognition and Brain Plasticity Unit, Department of Cognition, Development and Educational Psychology, University of \\ Barcelona and Bellvitge Biomedical Research Institute (IDIBELL), Barcelona, Spain. ${ }^{2}$ Department of Physical Medicine and \\ Rehabilitation, Hospitals del Mar i I'Esperança, Parc de Salut Mar, Barcelona, Spain. ${ }^{3}$ Neurophysiology Section, Department \\ of Neurology, Bellvitge University Hospital and Bellvitge Biomedical Research Institute (IDIBELL), Catalunya, Spain. \\ ${ }^{4}$ Cognitive Brain Research Unit, Department of Psychology and Logopedics, University of Helsinki, Helsinki, Finland. ${ }^{5}$ Catalan \\ Institution for Research and Advanced Studies (ICREA), Barcelona, Spain
}

Address for correspondence: Antoni Rodríguez-Fornells, Cognition and Brain Plasticity Unit, Group (IDIBELL), Department of Basic Psychology, University of Barcelona, Campus Bellvitge, Feixa Llarga, s/n (08907), L'Hospitalet de Llobregat, Barcelona, Spain. antoni.rodriguez@icrea.cat

The effect of music-supported therapy (MST) as a tool to restore hemiparesis of the upper extremity after a stroke has not been appropriately contrasted with conventional therapy. The aim of this trial was to test the effectiveness of adding MST to a standard rehabilitation program in subacute stroke patients. A randomized controlled trial was conducted in which patients were randomized to MST or conventional therapy in addition to the rehabilitation program. The intensity and duration of the interventions were equated in both groups. Before and after 4 weeks of treatment, motor and cognitive functions, mood, and quality of life (QoL) of participants were evaluated. A followup at 3 months was conducted to examine the retention of motor gains. Both groups significantly improved their motor function, and no differences between groups were found. The only difference between groups was observed in the language domain for QoL. Importantly, an association was encountered between the capacity to experience pleasure from music activities and the motor improvement in the MST group. MST as an add-on treatment showed no superiority to conventional therapies for motor recovery. Importantly, patient's intrinsic motivation to engage in musical activities was associated with better motor improvement.

Keywords: stroke; rehabilitation; music-supported therapy; randomized controlled trial

\section{Introduction}

Stroke is one of the main causes of death and long-term disability and is a significant burden on individuals and health care systems. ${ }^{1,2}$ Among other consequences, the majority of stroke survivors present paresis of the upper extremity. ${ }^{3}$ These deficits can lead to limitations in activities of daily life and restrictions in participation, thus decreasing the quality of life (QoL) of stroke patients. ${ }^{4,5}$

The recovery of motor deficits relies on rehabilitation programs, which are based on the principles of individualization, high intensity, and mass repetition of movements. ${ }^{6}$ These programs usually include physiotherapy and occupational therapy sessions on a daily basis during the first 6 months and up to a year following the stroke. ${ }^{7}$ In these sessions, a wide range of techniques are used, although a recent meta-analysis only supported the use of modified constraint-induced movement therapy and task-specific training in the first 4 weeks poststroke. ${ }^{8}$ In addition, other emerging evidencebased techniques could be introduced into the rehabilitation programs as add-on treatments to boost the recovery of stroke patients. ${ }^{9,10}$ These techniques are designed to improve not only motor deficits but 
also other relevant aspects, including motivation, treatment adherence, and mood, all of which are known to contribute to the success of the rehabilitation process. ${ }^{11,12}$ For instance, music practice as a therapy for stroke patients is an enjoyable activity that includes complex and coordinated movements while placing a high demand on cognitive functions, such as attention and working memory, as well as modulating mood. ${ }^{13-17}$ Music performance facilitates auditory-motor coupling, ${ }^{18}$ increases the adherence to physical exercises, ${ }^{19}$ and promotes social bonding during group performance. ${ }^{20-22}$ Some of these positive aspects could be partly mediated by the dopaminergic reward system, which is known to be highly responsive to music and involved in learning, memory, and the modulation of synaptic plasticity. ${ }^{23,24}$ Recently, optimization of motor learning has also been closely associated with intrinsic motivational factors. ${ }^{25}$ Both intensive motor training and motivational aspects could be effectively combined during music practice to improve motor recovery.

Specifically, music-supported therapy $(\mathrm{MST})^{26}$ aims to enhance the motor deficits of the upper extremity through the training with musical instruments and provides real-time auditory feedback about the performance, which serves as a basis for motor learning. ${ }^{27,28}$ Studies in the subacute and chronic stage of the rehabilitation process have demonstrated that stroke patients boost their motor function after the MST training. ${ }^{26,29-31} \mathrm{~A}$ 4-week program of MST can improve dexterity, smoothness, and velocity of movements and induce intrahemispheric functional reorganization within the lesioned hemisphere. ${ }^{18,32,33}$ MST targets motor deficits, but it can boost some aspects of cognition, mood, and QoL. ${ }^{18}$ Although the use of music-based therapies for the neurological population seems promising, high-quality randomized controlled trials (RCTs) are scarce. ${ }^{34}$ Moreover, it is still unclear whether adding MST to the conventional rehabilitation program can enhance motor recovery in subacute stroke patients. Furthermore, no previous study has directly compared the changes observed in emotional and QoL domains of subacute stroke patients treated with MST to standard treatment.

In this study, an RCT was designed with the aim of testing the effectiveness of adding MST to a program of conventional rehabilitation for subacute stroke patients. In contrast to previous studies with similar aims, ${ }^{26}$ in the present RCT, we controlled for the duration of the intervention provided to participants, making it equal in the experimental and the control groups. We hypothesized that patients treated with additional MST would show a major improvement in their upper extremity motor function compared with patients treated only with conventional therapies. We also expected an enhancement in other cognitive domains as well as in mood and QoL after receiving MST. Finally, and considering the importance of intrinsic motivation and engagement in the treatment success, ${ }^{25}$ we predicted that motor improvements in the MST group would be related to patient's individual differences in their capacity to experience pleasure in music activities.

\section{Methods}

\section{Study design}

A two-arm, parallel-group RCT was conducted in which a program of 4 weeks of MST was compared with conventional therapy in addition to a standard rehabilitation program. Before and after the 4-week treatment, the motor and cognitive functions as well as mood and QoL of the participants were evaluated. A follow-up evaluation of motor functions was also carried out at 3 months to assess the retention of gains. The study was approved by the ethical review board of the Hospital del Mar Medical Research Institute in Barcelona (registered trial at clinicaltrials.gov, ID: NCT02208219).

\section{Participants}

Subacute stroke patients involved in a program of outpatient rehabilitation at the Department of Physical Medicine and Rehabilitation at the Hospitals del Mar i de l'Esperança were assessed for recruitment from December 2013 to May 2017. Eligibility criteria were (1) mild-to-moderate paresis of the upper extremity after a first-ever stroke, (2) less than 6 months after the stroke, (3) age between 30 and 75 years, (4) no major cognitive deficits affecting comprehension (Mini-Mental State Examination ${ }^{35}$ $>24$ ), (5) no neurological or psychiatric comorbidity, (6) no previous formal musical education, and (7) ability to speak Spanish and/or Catalan. Mildto-moderate paresis was defined as having a score between 2 and 4 in the Medical Research Council Scale for Muscle Strength ${ }^{36}$ at the distal muscles of the upper extremity. 
The recruitment was performed by a medical doctor specialized in physical medicine and rehabilitation who provided information about the study in written and verbal format. Patients who agreed to participate signed an informed consent form.

\section{Randomization}

Participants were randomly allocated to a group receiving MST (MST group, $n=20$ ) or to a conventional therapy group (CT group, $n=20$ ) in addition to the standard program of rehabilitation. The randomization was stratified for laterality of the affected extremity. A statistician prepared a computer-generated random sequence, which was only accessible to one member of the research team who did not take part in the enrollment, evaluation, or treatment sessions. This researcher was responsible for informing the therapist about the group allocation of the participant at the beginning of the intervention.

\section{Treatment}

Both groups received an outpatient rehabilitation program that consisted of two 1-h group sessions of occupational therapy and physiotherapy a day ( 5 days per week, $10 \mathrm{~h}$ in total per week). During the occupational therapy sessions, functional and task-specific activities were trained to improve the motor performance of the affected upper extremity. In the physiotherapy sessions, patients exercised walking, balance, and global mobility. In addition to this standard program, participants were randomized into the MST or CT groups to receive 20 individual sessions ( 5 sessions per week, 30 min each) of MST in the MST group or extra time for exercises for the upper extremity in the CT group.

Participants in the MST group were trained to play a keyboard and an electronic drum set with the affected upper extremity following a modular therapy regime with stepwise increase of complexity. Participants in the CT group received individual training of the upper extremity, which included passive mobilization, stretch and progressive resistance exercises, and task-specific training. This group was conceived as an active control group (see the Appendix, online only, for a detailed explanation of exercises in the MST and CT groups). In both groups, the number of additional training hours was equal, and the sessions were administered individually by an occupational therapist.

\section{Evaluation of participants}

Demographic and clinical variables, such as age, gender, etiology of stroke, lesion location, date of stroke, and the scores of the National Health Institute Stroke Scale ${ }^{37}$ and the modified Rankin Scale ${ }^{38}$ at discharge of the stroke unit, were collected from medical records.

Primary outcome. The primary outcome was the functional movements of the paretic upper extremity measured with the action research arm test $(\mathrm{ARAT})^{39}$ at the end of the treatment.

\section{Secondary outcomes.}

Motor outcomes. Two measures of motor impairment were used: the upper extremity subtest of the Fugl-Meyer assessment ${ }^{40}$ and grip strength ${ }^{41}$ measured with a dynamometer as the mean of three trials. Fine dexterity was assessed with the ninehole pegboard test ${ }^{42}$ and box and blocks test. ${ }^{43}$ The functional use of the affected extremity in activities of daily living was evaluated with the Chedoke Arm and Hand Activity Inventory (CAHAI). ${ }^{44}$ The motor function evaluation was performed at baseline, after the intervention, and at a 3-month followup by an occupational therapist with experience in neurorehabilitation.

Cognitive outcomes. The cognitive evaluation was specifically focused on executive functions and memory. Working memory and attention were evaluated using the digit span (forward and backward) subtest from the Wechsler Adult Intelligence Scale III ${ }^{45}$ response inhibition by the Stroop task,${ }^{46}$ and processing speed and mental flexibility by the trailmaking test. ${ }^{47}$ Furthermore, the Rey auditory verbal learning test (RAVLT) ${ }^{48}$ and the story recall from the Rivermead behavioral memory test ${ }^{49}$ were both used to assess verbal memory. The cognitive assessment was performed at baseline and after the intervention by a neuropsychologist.

Mood and QoL outcomes. Mood was evaluated with the Profile of Mood States, ${ }^{50}$ the Beck Depression Inventory Scale, ${ }^{51}$ the Positive and Negative Affect Scale, ${ }^{52}$ and the Apathy Evaluation Scale. ${ }^{53}$ QoL was assessed with the Stroke Specific QoL Scale $^{54}$ and health-related QoL with the health survey questionnaire SF36. ${ }^{55}$ Questionnaires for mood and QoL were given to patients to be filled out at 
home before and after the treatment. The Barcelona Music Reward Questionnaire ${ }^{56}$ was completed by patients at baseline to evaluate individual differences in their capacity to experience pleasure from engaging in musical activities.

Participants also underwent an assessment with somatosensory evoked potentials and magnetic resonance imaging at the beginning and at the end of the treatment to obtain measurements of brain plasticity (structural and functional) as well as an evaluation of movement kinematics. This part of the study will be reported in future publications.

\section{Blinding}

All of the evaluators were blinded to participants' assigned treatment groups. Participants were inevitably aware of the treatment received, but they were asked not to inform the evaluators about the intervention, and no information about the specific differences between groups was provided to them.

\section{Statistical analysis}

Statistical analysis was performed using SPSS 21 software (SPSS Inc, Chicago, IL). A $t$-test for independent samples and the Pearson $\chi^{2}$ test were used for quantitative and nominal variables to test differences between groups at baseline. To test the effect of the intervention between groups, $t$-tests for independent samples were used for the change scores from baseline to posttreatment and from baseline to follow-up at 3 months. The within-group differences were tested using paired samples $t$-tests. The level of significance was set at 0.05 .

\section{Results}

\section{Demographic and clinical characteristics}

A total of 178 stroke patients receiving an outpatient rehabilitation program in the recruitment site were assessed for eligibility (Fig. 1). Among them, 129 did not meet the inclusion criteria, and 9 declined to participate. Of the 40 patients enrolled in the study, 20 were assigned to the MST group and 20 to the CT group. In the MST group, one patient was transferred to another clinic after randomization. There were no significant differences in demographic and clinical variables between groups at baseline, as outlined in Table 1, except for the variables etiology of stroke and number of participants with cortical lesions. The CT group had a major distribution of hemorrhagic strokes compared with the MST group, and the number of participants who had lesions that involved cortical regions was higher in the MST group than in the CT group (for a detailed clinical description of participants, see Fig. S1 and Table S1, online only). The number of hours of rehabilitation that patients received before enrollment was equal between groups. Importantly, there were no significant differences between groups at baseline in any of the assessments used for primary and secondary outcomes.

At the follow-up evaluation at 3 months, three participants in the MST group (two unable to contact and one withdraw) and two participants (unable to contact) in the CT group dropped out. With regard to the secondary outcomes, some participants could not complete all the questionnaires at the posttreatment evaluation owing to fatigue but were included in the study, as they underwent most of the evaluation. The exact number of participants per test is provided in Tables S2-S5 (online only).

\section{Primary outcome}

For the overall score of the ARAT, no differences in change scores were found between groups at posttreatment and 3-month follow-up (Fig. 2 and Table S2, online only). Both groups significantly improved after the intervention. The MST group had a mean improvement of $9.8( \pm 7.9)$ points after the treatment $(t(18)=-5.37, P<0.001, d=1.23)$ and $12.4( \pm 14)$ at the follow-up evaluation $(t(15)=$ $-3.55, P<0.005, d=0.89)$. The CT group improved $6.7( \pm 7.9)$ points after the treatment $(t(19)=-3.75$, $P=0.001, d=0.84)$ and $7.3( \pm 8.1)$ at the follow-up performed at 3 months $(t(17)=-3.82, P=0.001$, $d=0.90$ ). The minimal detectable change (MDC) for this test is a score of 5.7 points; and 14 of 19 participants in the MST group reached the MDC level after treatment, and 11 out of 16 did it at 3 months. In the CT group, 9 of 20 participants presented a change that was clinically relevant after treatment, and 9 out of 18 reached the MDC level at 3 months (Fig. 2). The difference between groups in the number of patients who reached the MDC was not significant $\left(\chi^{2}(1)=3.31, P=0.069\right.$ for posttreatment and $\chi^{2}(1)=1.22, P=0.268$ for follow-up).

\section{Secondary outcomes}

Regarding the secondary motor outcomes, no significant differences were observed for the group comparisons after treatment or at follow-up (Fig. 2 and Table S2, online only). The within-group analyses revealed that both groups improved after 


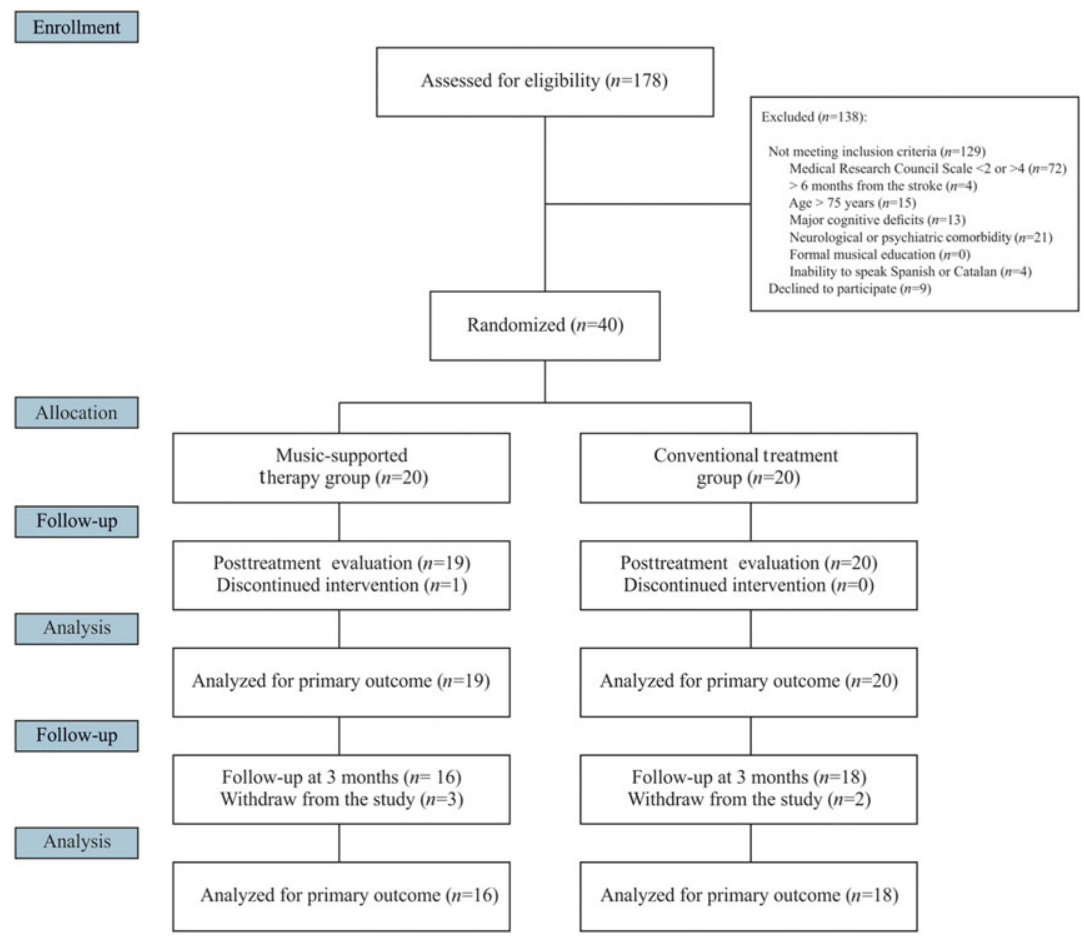

Figure 1. Study design and participant flow. Flow diagram with the phases of the two-arm, parallel randomized controlled trial and the number of participants per group in each of the phases.

treatment and at follow-up in the Fugl-Meyer assessment, nine-hole pegboard test, box and blocks test, and the CAHAI (Table S2, online only).

For the cognitive outcomes, groups did not differ in the change scores from baseline to posttreatment (Table S3, online only). However, when testing the within-group differences, participants in the MST group improved in verbal learning of the RAVLT $(t(17)=-2.67, P<0.05, d=0.97)$.

For the mood and QoL outcomes, the change score of the language domain of the Stroke Specific QoL Scale was significantly different between groups $(t(35)=2.96, P=0.005, d=0.20)$ (Fig. 3 and Tables S4 and S5, online only). The MST group improved in the language domain from baseline to posttreatment $(t(18)=-2.23, P<0.05$, $d=0.54$ ), while the CT group did not show any improvement. There were no significant differences between groups in the change scores of the other tests assessing mood and QoL. The within-groups analyses in the MST group showed a reduction in the fatigue-inertia component of the Profile of Mood States $(t(16)=2.45, P<0.05, d=0.62)$ and the negative affect measured with the Positive and Negative
Affect Scale after the treatment $(t(16)=2.25, P<$ $0.05, d=0.64$ ) (Fig. 3). In this group, the overall score of the Stroke Specific QoL Scale $(t(18)=-2.66$, $P<0.05, d=0.64)$, as well as the self-care $(t(18)=$ $-3.44, P<0.005, d=0.80)$ and productivity domains $(t(18)=-3.57, P<0.005, d=0.83)$ improved after the therapy. Patients in the MST group also presented an improvement in the physical and social function components of the SF-36 Health-related QoL $(t(16)=-2.24, P<0.05, d=$ $0.54, t(16)=-3.70, P<0.005, d=0.90)$ (Fig. 3). In the CT group, only a significant difference was found for the self-care domain of the Stroke Specific QoL after the treatment $(t(17)=-3.95, P=0.001$, $d=1.04$ ).

\section{Music reward experiences and response to MST}

A positive correlation was found between the improvement in ARAT at 3 months and the sensorymotor component of the Barcelona Music Reward Questionnaire for the MST group $(r=0.562, P=$ $0.024)$ but not for the CT group $(r=-0.305$, $P<0.21$ ) (Fig. 2). Patients in the MST group with 
Table 1. Demographic and clinical variables for both groups at baseline

\begin{tabular}{|c|c|c|c|}
\hline & $\begin{array}{l}\text { MST group } \\
\quad(n=19)\end{array}$ & $\begin{array}{l}\text { CT group } \\
(n=20)\end{array}$ & $P$ value \\
\hline \multicolumn{4}{|l|}{ Demographic variables } \\
\hline Age (range) & $60.1(45-74)$ & $62.5(49-72)$ & 0.314 \\
\hline \multicolumn{4}{|l|}{ Gender } \\
\hline Females (\%) & $8(42.1)$ & $8(40)$ & \multirow[t]{2}{*}{0.894} \\
\hline Males (\%) & $11(57.9)$ & $12(60)$ & \\
\hline \multicolumn{4}{|l|}{ Clinical variables } \\
\hline \multicolumn{4}{|l|}{ Stroke etiology } \\
\hline Ischemic (\%) & $18(94.7)$ & $14(70)$ & \multirow[t]{2}{*}{$0.044^{*}$} \\
\hline Hemorrhagic (\%) & $1(5.3)$ & $6(30)$ & \\
\hline \multicolumn{4}{|l|}{ Lesion location } \\
\hline Cortical (\%) & $7(36.8)$ & $1(5)$ & $0.014^{*}$ \\
\hline Subcortical (\%) & $16(84.2)$ & $16(80)$ & 0.732 \\
\hline Brainstem (\%) & $2(10.5)$ & $3(15)$ & 0.676 \\
\hline Cerebellum (\%) & $1(5.3)$ & $0(0)$ & 0.299 \\
\hline Time since stroke (range) & $65.8(32-162)$ & $64.9(28-136)$ & 0.085 \\
\hline NHISS (range) & $5.8(2-14)$ & $5.3(2-9)$ & 0.434 \\
\hline Modified Rankin Scale (range) & $3.5(3-4)$ & $3.2(2-4)$ & 0.237 \\
\hline Fugl-Meyer baseline (range) & $45.3(24-65)$ & $45.8(21-65)$ & 0.904 \\
\hline \multicolumn{4}{|l|}{ Rehabilitation received before enrollment } \\
\hline Physiotherapy & $26.6(20.4)$ & $17(12.1)$ & 0.088 \\
\hline Occupational therapy & $23.5(19.7)$ & $19.1(16)$ & 0.452 \\
\hline Speech therapy & $14.2(12.4)$ & $12.2(5.9)$ & 0.784 \\
\hline \multicolumn{4}{|l|}{ Reward in musical activities } \\
\hline Barcelona Music Reward Questionnaire & $73.9(10.6)$ & $71.8(13.6)$ & 0.594 \\
\hline
\end{tabular}

Noте: Absolute frequencies are shown for the variables gender, stroke etiology, and lesion location. For the rest of variables, the mean and standard deviation are shown, unless indicated otherwise. The differences between groups were evaluated with $t$-tests for independent samples for quantitative variables and the Pearson $\chi^{2}$ test for nominal ones.

${ }^{*} P<0.05$.

higher capacity to experience pleasure from musical activities (sensorimotor component) showed a major improvement in the ARAT at 3 months. Significant differences were observed between both correlations using the Fisher's $z$ test $(z=2.4, P<0.05)$.

\section{Discussion}

In this RCT, we examined the effectiveness of adding MST to a rehabilitation program for subacute stroke patients and compared it to standard rehabilitatio alone after controlling for the total duration of the intervention. Regarding our primary and secondary motor outcomes, no group differences were observed at the end of the treatment or at the 3-month follow-up (Fig. 2). With regard to the mood and QoL secondary outcomes, a larger improvement in the MST group was found for the language domain of the Stroke Specific QoL Scale after treatment when compared with the CT group. Importantly, in the MST group, a significant association was encountered between patients' individual differences in their capacity to experience pleasure from engaging in musical activities and motor improvement (primary outcome at 3month follow-up) (Fig. 2). This association was not present in the CT group.

No superiority effect in adding MST to a standard program of rehabilitation at the motor level was observed in the present trial. Both groups received the same standard program, and, in the CT group, additional training was provided to compensate for the extra time that the MST group received. As expected in the subacute stage, patients in both groups improved their motor deficits, showing an enhancement in dexterity, functional movements, and motor performance in activities of daily living. Our results differ from the study of Schneider and colleagues, ${ }^{26}$ in which patients receiving 


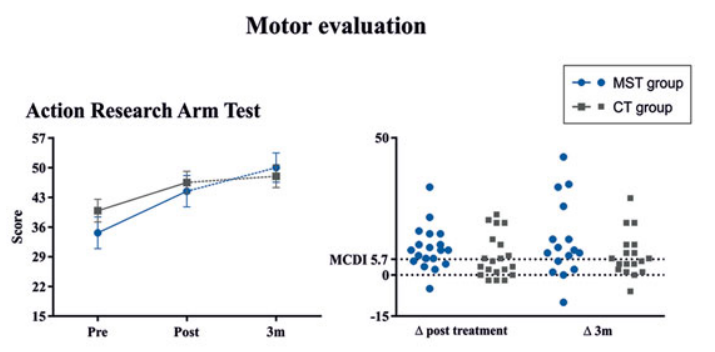

Fugl-Meyer Assessment
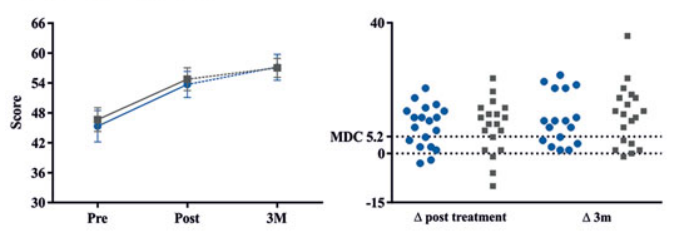

Grip Strength
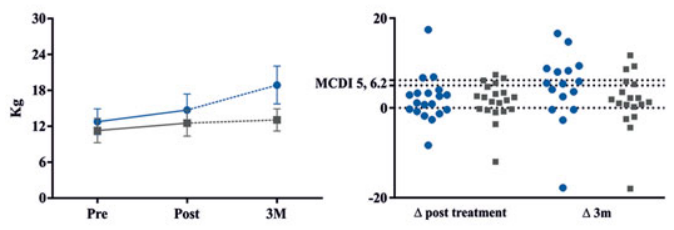

Chedoke Arm and Hand Activity Inventory

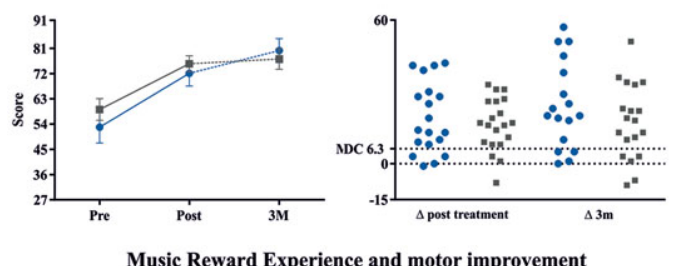

Music Reward Experience and motor improvement

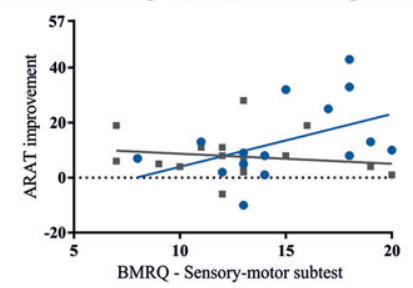

Figure 2. Results for the primary and secondary motor outcomes. Results for the action research arm test (ARAT), the Fugl-Meyer assessment, the grip strength, and the Chedoke Arm and Hand Activity Inventory at baseline (pre), after the intervention (post) and at the 3 months followup (3 months) are shown for both groups. For each test, the improvements from baseline to posttreatment ( $\Delta$ posttreatment) and from baseline to the follow-up at 3 months ( $\Delta 3$ months) are shown for both groups. The dotted lines indicate the threshold of the minimal detectable change (MDC) or minimally clinically important difference (MCID) of each test. The correlation between the sensorimotor component of the Barcelona Music Reward Questionnaire (BMRQ) and the improvement in the ARAT at 3 months is shown for both groups.
MST in addition to other standard rehabilitation techniques increased the speed and smoothness of movements as well as their overall motor function when compared with a control group. In this study, the experimental and control group received around 13.5 h of conventional rehabilitation within 3 weeks. In addition, the experimental group spent $30 \mathrm{~min}$ more per day (3 weeks) undergoing MST, but no extra rehabilitation time was provided to the control group. In our study, groups received nearly $40 \mathrm{~h}$ of conventional rehabilitation during 4 weeks, and both the MST and CT groups were provided $30 \mathrm{~min}$ more per day of MST or extra training with conventional therapies. Whereas the improvement of the MST group is similar in both studies, the training intensity of the control group might explain the different results obtained in the two studies. An important aspect to consider for future trials is to what extent the MST program might show larger effects when added to standard rehabilitation programs of shorter duration and intensity. MST could boost motor improvement when fewer resources could be devoted to individualized conventional physiotherapy and occupational therapy programs after subacute stroke.

An outstanding finding in our study is that, in the MST group, patients' intrinsic motivation to participate and enjoy musical activities (and in particular musical activities involving a strong sensorimotor component (e.g., tapping to the beat, humming, singing, or dancing)) correlated with a larger improvement in the primary motor outcome at 3 months when compared with the CT group. This result agrees with recent proposals in the field of motor learning highlighting the importance of motivational factors in motor skill learning. ${ }^{25}$ Moreover, recent studies have highlighted the significant role of intrinsic motivation in successful learning, an effect mediated by midbrain dopaminergic pathways and their interaction with reward-memory circuits. ${ }^{57-59}$ MST training is adapted to patient's needs, providing exercises that challenge the patient but at the same time are achievable, promoting autonomy and giving patients control over the learning experience. This might reduce the perceived task difficulty, distracting attention from the effort required, ${ }^{60}$ and increase feelings of self-efficacy. ${ }^{25}$ At the same time, during the MST sessions, the therapist provides direct positive feedback about the patient's performance, 


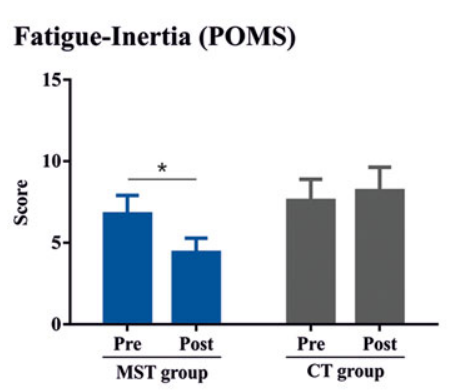

Mood and Quality of Life
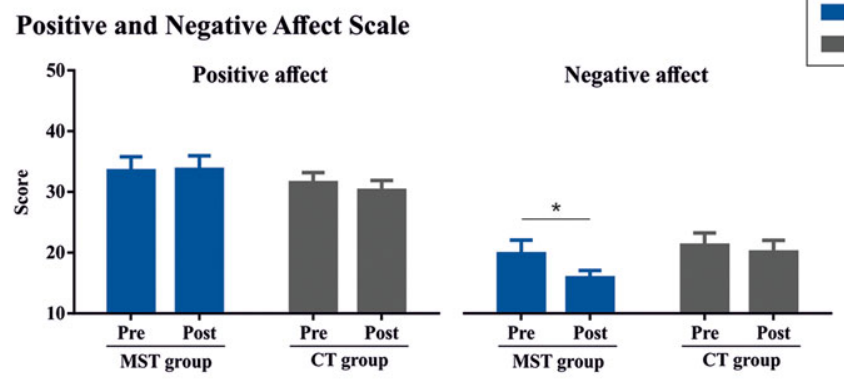

Apathy evaluation Scale
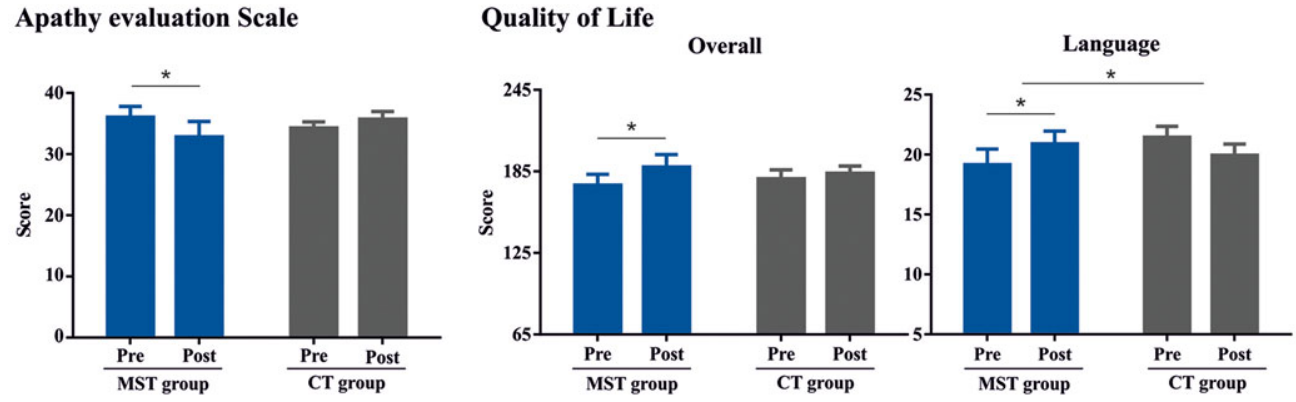

Figure 3. Results for the secondary mood and quality of life outcomes. Results for the fatigue-inertia component of the Profile of Mood States (POMS), the Positive and Negative Affect Scale, the Apathy Evaluation Scale, and the Stroke Specific Quality of life Scale (overall and language domain) are shown for both groups. ${ }^{\star} P<0.05$.

reinforcing the learning experience. Taken together, these motivational aspects could influence motor skill learning in patients with higher reward sensitivity to music. ${ }^{61,62}$

Although no difference was observed in the improvement at emotional level between the two groups, it is relevant to note that within-group analysis in the MST group revealed a significant reduction in fatigue and negative affect and a better QoL after treatment. The CT group only improved in the self-care QoL domain. Additionally, although there were no differences between groups at the cognitive level, the within-groups analyses showed that the MST group improved their attention and verbal memory after the training. Similar findings have been previously described at the cognitive and emotional levels after MST in chronic stroke patients, ${ }^{18,30}$ as well as in other music programs for neurological population. ${ }^{10,13,63,64}$ Thus, these results reinforce the capacity of music in therapeutic programs as a mood enhancer. ${ }^{65}$

The potential impact of music in stroke recovery fits with the recent findings of the EVREST trial, in which it was observed that recreational activities can be as effective as other sophisticated motor rehabil- itation programs. ${ }^{66}$ Future studies should consider the intensity and duration of the standard rehabilitation program to which the MST is added, as well as the dose-response of MST. ${ }^{67}$ Moreover, aspects influencing the overall recovery process, such as the lesion location, white matter damage, and the extent of deficits, should be taken into account. The MST program could be modified by introducing new components, such as the self-selection of songs or musical styles, improvization, music sonification, ${ }^{68}$ and other types of feedback that are in line with the most advanced motor learning paradigms. MST can also be adapted in order to be applied at home or in groups to incorporate more social aspects. ${ }^{69,70}$

In this study, MST in addition to the rehabilitation program did not show superiority over conventional therapies. Importantly, the patient's intrinsic motivation to engage in music activities should be taken into account, since we observed an association between motivation and motor improvement. In this sense, new questions arise in the design of more individualized rehabilitation programs, tailoring the activities or therapies provided in the rehabilitation centers to the interest of the patient. 


\section{Acknowledgments}

The authors want to thank Patricia León, David Cucurell, Xavier Rifà, Lucía Canitrot, and Erin McDonough for all their help and Dr. Anna Guillén and Dr. Roser Boza for their support throughout the entire study. The authors are also very grateful to the patients for taking part in the study. The research leading to these results has received funding from RecerCaixa. This work was supported by the Spanish Government (Ministerio de Economía y Competitivdad, PSI2015-69178-P, Fondo Europeo de Desarrollo Regional (FEDER) "Una manera de hacer Europa" / "a way to build Europe”).

\section{Supporting Information}

Additional supporting information may be found in the online version of this article.

Appendix. Description of treatments.

Figure S1. Lesion location of participants.

Table S1. Description of participants.

Table S2. Results for the motor primary and secondary outcomes.

Table S3. Results for the cognitive secondary outcomes.

Table S4. Results for the mood secondary outcomes.

Table S5. Results for the quality of life secondary outcomes.

\section{Competing interests}

The authors declare no competing interests.

\section{References}

1. Feigin, V.L., G.A. Mensah, B. Norrving, et al. 2015. Atlas of the global burden of stroke (1990-2013): the GBD 2013 study. Neuroepidemiology 45: 230-236.

2. Murray, C.J.L., T. Vos, R. Lozano, et al. 2012. Disabilityadjusted life years (DALYs) for 291 diseases and injuries in 21 regions, 1990-2010: a systematic analysis for the Global Burden of Disease Study 2010. Lancet 380: 21972223.

3. Rathore, S.S., A.R. Hinn, L.S. Cooper, et al. 2002. Characterization of incident stroke signs and symptoms findings from the atherosclerosis risk in communities study. Stroke 33: 2718-2721.

4. Mayo, N.E., S. Wood-Dauphinee, R. Côté, et al. 2002. Activity, participation, and quality of life 6 months poststroke. Arch. Phys. Med. Rehabil. 83: 1035-1042.

5. Mayo, N.E., D. Bronstein, S.C. Scott, et al. 2014. Necessary and sufficient causes of participation post-stroke: practi- cal and philosophical perspectives. Qual. Life Res. 23: 3947.

6. Albert, S.J. \& J. Kesselring. 2012. Neurorehabilitation of stroke. J. Neurol. 259: 817-832.

7. Langhorne, P., J. Bernhardt \& G. Kwakkel. 2011. Stroke rehabilitation. Lancet 377: 1693-1702.

8. Wattchow, K.A., M.N. McDonnell \& S.L. Hillier. 2017. Rehabilitation interventions for upper limb function in the first four weeks following stroke: a systematic review and meta-analysis of the evidence. Arch. Phys. Med. Rehabil. https://doi.org/10.1016/j.apmr.2017.06.014.

9. Hatem, S.M., G. Saussez, M. della Faille, et al. 2016. Rehabilitation of motor function after stroke: a multiple systematic review focused on techniques to stimulate upper extremity recovery. Front. Hum. Neurosci. 10: 442.

10. François, C., J. Grau-Sánchez, E. Duarte, et al. 2015. Musical training as an alternative and effective method for neuroeducation and neuro-rehabilitation. Front. Psychol. 6: 115.

11. Luker, J., E. Lynch, S. Bernhardsson, et al. 2015. Stroke survivors' experiences of physical rehabilitation: a systematic review of qualitative studies. Arch. Phys. Med. Rehabil. 96: 1698-1708.e10.

12. Poltawski, L., K. Boddy, A. Forster, et al. 2015. Motivators for uptake and maintenance of exercise: perceptions of longterm stroke survivors and implications for design of exercise programmes. Disabil. Rehabil. 37: 795-801.

13. Sihvonen, A.J., T. Särkämö, V. Leo, et al. 2017. Music-based interventions in neurological rehabilitation. Lancet Neurol. 16: 648-660.

14. Raghavan, P., D. Geller, N. Guerrero, et al. 2016. Music upper limb therapy-integrated: an enriched collaborative approach for stroke rehabilitation. Front. Hum. Neurosci. 10: 498.

15. Raglio, A., A. Zaliani, P. Baiardi, et al. 2017. Active music therapy approach for stroke patients in the post-acute rehabilitation. Neurol. Sci. 38: 893-897.

16. Alves-Pinto, A., V. Turova, T. Blumenstein, et al. 2016. The case for musical instrument training in cerebral palsy for neurorehabilitation. Neural. Plast. 2016: 1072301.

17. Lampe, R., A. Thienel, J. Mitternacht, et al. 2015. Piano training in youths with hand motor impairments after damage to the developing brain. Neuropsychiatr. Dis. Treat. 11: 1929-1938.

18. Ripollés, P., N. Rojo, J. Grau-Sánchez, et al. 2016. Music supported therapy promotes motor plasticity in individuals with chronic stroke. Brain Imaging Behav. 10: 12891307.

19. Wininger, S.R. \& D. Pargman. 2003. Assessment of factors associated with exercise enjoyment. J. Music Ther. 40: 57-73.

20. Cirelli, L.K., K.M. Einarson \& L.J. Trainor. 2014. Interpersonal synchrony increases prosocial behavior in infants. Dev. Sci. 17: 1003-1011.

21. Hove, M.J. \& J.L. Risen. 2009. It's all in the timing: interpersonal synchrony increases affiliation. Soc. Cogn. 27: 949960.

22. Guerrero, N., A. Turry, D. Geller, et al. 2014. From historic to contemporary: Nordoff-Robbins music therapy in collaborative interdisciplinary rehabilitation. Music Ther. Perspect. 32: $38-46$. 
23. Lisman, J.E., A.A. Grace, T. Nakajima, et al. 2005. The hippocampal-VTA loop: controlling the entry of information into long-term memory. Neuron 46: 703-713.

24. Adcock, R.A., A. Thangavel, S. Whitfield-Gabrieli, et al. 2006. Reward-motivated learning: mesolimbic activation precedes memory formation. Neuron 50: 507-517.

25. Wulf, G. \& R. Lewthwaite. 2016. Optimizing performance through intrinsic motivation and attention for learning: the OPTIMAL theory of motor learning. Psychon. Bull. Rev. 23: 1382-1414.

26. Schneider, S., P.W. Schönle, E. Altenmüller, et al. 2007. Using musical instruments to improve motor skill recovery following a stroke. J. Neurol. 254: 1339-1346.

27. Rodriguez-Fornells, A., N. Rojo, J.L. Amengual, et al. 2012. The involvement of audio-motor coupling in the musicsupported therapy applied to stroke patients. Ann. N.Y. Acad. Sci. 1252: 282-293.

28. Zatorre, R.J., J.L. Chen \& V.B. Penhune. 2007. When the brain plays music: auditory-motor interactions in music perception and production. Nat. Rev. Neurosci. 8: 547558.

29. Altenmüller, E., J. Marco-Pallares, T.F. Münte, et al. 2009. Neural reorganization underlies improvement in strokeinduced motor dysfunction by music-supported therapy. Ann. N.Y. Acad. Sci. 1169: 395-405.

30. Rojo, N., J. Amengual, M. Juncadella, et al. 2011. Musicsupported therapy induces plasticity in the sensorimotor cortex in chronic stroke: a single-case study using multimodal imaging (fMRI-TMS). Brain Inj. 25: 787793.

31. Tong, Y., B. Forreider, X. Sun, et al. 2015. Music-supported therapy (MST) in improving post-stroke patients' upperlimb motor function: a randomised controlled pilot study. Neurol. Res. 37: 434-440.

32. Amengual, J.L., N. Rojo, M. Veciana de las Heras, et al. 2013. Sensorimotor plasticity after music-supported therapy in chronic stroke patients revealed by transcranial magnetic stimulation. PLoS One 8: e61883.

33. Grau-Sánchez, J., J.L. Amengual, N. Rojo, et al. 2013. Plasticity in the sensorimotor cortex induced by music-supported therapy in stroke patients: a TMS study. Front. Hum. Neurosci. 7: 494.

34. Magee, W.L., I. Clark, J. Tamplin \& J. Bradt. 2017. Music interventions for acquired brain injury. Cochrane Database Syst. Rev. 1: CD006787.

35. Folstein, M.F., S.E. Folstein \& P.R. McHugh. 1975. Minimental state. A practical method for grading the cognitive state of patients for the clinician. J. Psychiatr. Res. 12: 189198.

36. Medical Research Council. 1981. Aids to the examination of the peripheral nervous system, Memorandum No. 45. London: Her Majesty's Stationery Office.

37. Brott, T., H.P. Adams, Jr, C.P. Olinger, et al. 1989. Measurements of acute cerebral infarction: a clinical examination scale. Stroke 20: 864-870.

38. van Swieten, J.C., P.J. Koudstaal, M.C. Visser, et al. 1988. Interobserver agreement for the assessment of handicap in stroke patients. Stroke 19: 604-607.
39. Lyle, R.C. 1981. A performance test for assessment of upper limb function in physical rehabilitation treatment and research. Int. J. Rehabil. Res. 4: 483-492.

40. Fugl-Meyer, A.R., L. Jääskö, I. Leyman, et al. 1975. The post-stroke hemiplegic patient. 1. A method for evaluation of physical performance. Scand. J. Rehabil. Med. 7: 13-31.

41. Mathiowetz, V., K. Weber, G. Volland, et al. 1984. Reliability and validity of grip and pinch strength evaluations. J. Hand Surg. Am. 9: 222-226.

42. Parker, V.M., D.T. Wade \& R. Langton Hewer. 1986. Loss of arm function after stroke: measurement, frequency, and recovery. Int. Rehabil. Med. 8: 69-73.

43. Mathiowetz, V., G. Volland, N. Kashman, et al. 1985. Adult norms for the Box and Block Test of manual dexterity. Am. J. Occup. Ther. 39: 386-391.

44. Barreca, S., C.K. Gowland, P. Stratford, et al. 2004. Development of the Chedoke Arm and Hand Activity Inventory: theoretical constructs, item generation, and selection. Top. Stroke Rehabil. 11: 31-42.

45. Wechsler, D. 1939. The Measurement of Adult Intelligence. Baltimore, MD: Williams \& Wilkins.

46. Stroop, J.R. 1935. Studies of interference in serial verbal reactions. J. Exp. Psychol. 18: 643-662.

47. Reitan, R.M. 1958. Validity of the trail making test as an indicator of organic brain damage. Percept. Mot. Skills 8: 271-276.

48. Rey, A. 1964. L'examen Clinique en Psychologie. 2nd ed. Paris: Presses Universitaires de France.

49. Wilson, B.A. 2008. The Rivermead Behavioural Memory Test-Third Edition RBMT 3; Administration and Scoring Manual. Pearson.

50. McNair, D., M. Lorr \& L. Droppleman. 1981. Edits Manual for the Profile of Mood States. San Diego, CA: Educational and Industrial Testing Services.

51. Beck, A.T., C.H. Ward, M. Mendelson, et al. 1961. An inventory for measuring depression. Arch. Gen. Psychiatry 4: 561571.

52. Watson, D., L.A. Clark \& A. Tellegen. 1988. Development and validation of brief measures of positive and negative affect: the PANAS scales. J. Pers. Soc. Psychol. 54: 1063-1070.

53. Marin, R.S., R.C. Biedrzycki, S. Firinciogullari, et al. 1991. Reliability and validity of the Apathy Evaluation Scale. Psychiatry Res. 38: 143-162.

54. Williams, L.S., M. Weinberger, L.E. Harris, et al. 1999. Development of a Stroke-Specific Quality of Life Scale. Stroke 30: 1362-1369.

55. Alonso, J., L. Prieto \& J.M. Antó. 1995. [The Spanish version of the SF-36 Health Survey (the SF-36 health questionnaire): an instrument for measuring clinical results]. Med.Clin. 104: 771-776.

56. Mas-Herrero, E., J. Marco-Pallares, U. Lorenzo-Seva, et al. 2013. Individual differences in music reward experiences. Music Percept. 31: 118-138.

57. Gruber, M.J., B.D. Gelman \& C. Ranganath. 2014. States of curiosity modulate hippocampus-dependent learning via the dopaminergic circuit. Neuron 84: 486-496.

58. Ripollés, P., J. Marco-Pallarés, U. Hielscher, et al. 2014. The role of reward in word learning and its implications for language acquisition. Curr. Biol. 24: 2606-2611. 
59. Ripollés, P., J. Marco-Pallarés, H. Alicart, et al. 2016. Intrinsic monitoring of learning success facilitates memory encoding via the activation of the SN/VTA-Hippocampal loop. Elife 5. https://doi.org/10.7554/eLife.17441.

60. Dyrlund, A.K. \& S.R. Wininger. 2008. The effects of music preference and exercise intensity on psychological variables. J. Music Ther. 45: 114-134.

61. Dayan, E. \& L.G. Cohen. 2011. Neuroplasticity subserving motor skill learning. Neuron 72: 443-454.

62. Krakauer, J.W. 2006. Motor learning: its relevance to stroke recovery and neurorehabilitation. Curr. Opin. Neurol. 19: 84-90.

63. Särkämö, T., M. Tervaniemi, S. Laitinen, et al. 2008. Music listening enhances cognitive recovery and mood after middle cerebral artery stroke. Brain 131: 866-876.

64. Särkämö, T., M. Tervaniemi, S. Laitinen, et al. 2014. Cognitive, emotional, and social benefits of regular musical activities in early dementia: randomized controlled study. Gerontologist 54: 634-650.

65. Altenmüller, E. \& G. Schlaug. 2015. Apollo's gift: new aspects of neurologic music therapy. Prog. Brain Res. 217: 237-252.
66. Saposnik, G., L.G. Cohen, M. Mamdani, et al. 2016. Efficacy and safety of non-immersive virtual reality exercising in stroke rehabilitation (EVREST): a randomised, multicentre, single-blind, controlled trial. Lancet Neurol. 15: 10191027.

67. Grau-Sánchez, J., N. Ramos, E. Duarte, et al. 2017. Time course of motor gains induced by music-supported therapy after stroke: an exploratory case study. Neuropsychology 31: 624-635.

68. Scholz, D.S., S. Rohde, N. Nikmaram, et al. 2016. Sonification of arm movements in stroke rehabilitation-a novel approach in neurologic music therapy. Front. Neurol. 7: 106.

69. Villeneuve, M., V. Penhune \& A. Lamontagne. 2014. A piano training program to improve manual dexterity and upper extremity function in chronic stroke survivors. Front. Hum. Neurosci. 8: 662.

70. Street, A.J., W.L. Magee, H. Odell-Miller, et al. 2015. Homebased neurologic music therapy for upper limb rehabilitation with stroke patients at community rehabilitation stage-a feasibility study protocol. Front. Hum. Neurosci. 9: 480 . 